PROCEEDINGS OF THE AMERICAN MATHEMATICAL SOCIETY

Volume 125, Number 3, March 1997, Pages 927-934

S 0002-9939(97)04030-6

\title{
FROM COUNTABLE COMPACTNESS TO ABSOLUTE COUNTABLE COMPACTNESS
}

\author{
MARY ELLEN RUDIN, IAN S. STARES, AND JERRY E. VAUGHAN \\ (Communicated by Franklin D. Tall)
}

\begin{abstract}
We show that every countably compact space which is monotonically normal, almost 2 -fully normal, radial $T_{2}$, or $T_{3}$ with countable spread is absolutely countably compact. For the first two mentioned properties, we prove more general results not requiring countable compactness. We also prove that every monotonically normal, orthocompact space is finitely fully normal.
\end{abstract}

\section{INTRODUCTION}

M. V. Matveev [13] defined a space $X$ to be absolutely countably compact (acc) provided for every open cover $\mathcal{U}$ of $X$ and every dense $D \subseteq X$, there exists a finite set $F \subseteq D$ such that $S t(F, \mathcal{U})=X$. He noted that every compact space is acc, and in the class of $T_{2}$-spaces every acc space is countably compact. Several classes are known wherein every countably compact space is absolutely countably compact. For example, every countably compact space of countable tightness is acc [13], and every countably compact, orthocompact space is acc [14].

In this paper we give four more classes of spaces in which countably compact spaces are absolutely countably compact: the classes of monotonically normal spaces (Theorem 1), almost 2-fully normal spaces (Theorem 3 ), radial $T_{2}$-spaces (Theorem 4 ), and $T_{3}$-spaces having countable spread (Theorem 5 ) (this last result follows immediately from two known results). Two of these results answer questions from [14]. Still open is whether every normal, countably compact space is acc (see [13, Question 1.12] and [14, Question 1.11]).

In E-mail correspondence with the authors, Matveev defined the following property:

Definition 1 (M. Matveev). A space $X$ has property (a) provided for every open cover $\mathcal{U}$ of $X$ and every dense $D \subseteq X$, there exists a set $F \subseteq D$ such that $F$ is discrete in $X$ (i.e., $F$ is closed in $X$ and a discrete subspace of $X$ ) and $S t(F, \mathcal{U})=X$.

Clearly the motivation for property (a) is that every countably compact space with property (a) is acc. Matveev raised the question: Does every monotonically

Received by the editors September 10, 1995.

1991 Mathematics Subject Classification. Primary 54D20; Secondary 54A35.

Key words and phrases. Countably compact, absolutely countably compact, monotonically normal, property (a), finitely fully normal, almost 2-fully normal, radial, orthocompact, countable spread. 
normal space have property (a)? We give an affirmative answer to Matveev's question in Theorem 1, and we also show that almost 2-full normality implies (a). Radial spaces, however, do not have property (a) in general.

We now give several more definitions used in this paper. For terms not defined, see $[9]$.

A space $X$ is called radial (cf. [2, p. 42]) provided for every $A \subseteq X$ and every $x \in \bar{A}$, there exists a regular infinite cardinal $\kappa$ and a transfinite sequence $f: \kappa \rightarrow A$ such that $f$ converges to $x$.

The $\aleph_{0}$-closure ([1]) of a subset $D$ of $X$ is defined to be

$$
[D]_{\aleph_{0}}=\left\{x \in X: \exists H \in[D]^{\omega} \text { such that } x \in \bar{H}\right\} .
$$

A $T_{1}$-space $X$ is said to be monotonically normal if there is an operator $H$ which assigns to each ordered pair $(A, U)$, where $A$ is closed, $U$ is open and $A \subseteq U$, an open set $H(A, U)$ such that

(MN1): $A \subseteq H(A, U) \subseteq \overline{H(A, U)} \subseteq U$,

(MN2): if $A \subseteq B$ and $U \subseteq V$, then $H(A, U) \subseteq H(B, V)$,

(MN3): if $H(\{x\}, U) \cap H(\{y\}, V) \neq \emptyset$, then either $x \in V$ or $y \in U$.

Although not the original definition of monotone normality (see [11]) this definition is equivalent to the usual one [7]. We shall abuse notation and write $H(x, U)$ for $H(\{x\}, U)$.

A space $X$ is said to be almost 2-fully normal [12] if for every open cover $\mathcal{U}$ of $X$ there is a refinement $\mathcal{V}$ of $\mathcal{U}$ (known as an almost 2-star refinement) such that if $x \in X$ and $y, z \in S t(x, \mathcal{V})$ then there is $U \in \mathcal{U}$ such that $y, z \in U$. Van Douwen [8, p. 84] referred to almost 2-full normality as "... the weakest known covering property which implies collectionwise normality".

A space $X$ is said to have countable spread provided every discrete subspace of $X$ is at most countable.

\section{TWO PROPERTIES IMPLYING (a)}

The following answers Question 1.12 in [14].

Theorem 1. Monotonically normal spaces satisfy (a). In particular, every monotonically normal, countably compact space is acc.

Our proof of Theorem 1 uses the following well-known result.

Theorem 2 (Z. Balogh and M. E. Rudin [4]). If $X$ is monotonically normal and $\mathcal{U}$ is an open cover of $X$, then there is a $\sigma$-disjoint open partial refinement $\mathcal{V}$ of $\mathcal{U}$ such that $X \backslash \cup \mathcal{V}$ is the union of a closed discrete collection $\mathcal{Y}$ of copies of stationary subsets of regular uncountable ordinals.

Proof of Theorem 1. Suppose that $X$ is monotonically normal, $\mathcal{U}$ is an open cover of $X$ and $D$ is a dense subset of $X$. Let $H$ be a monotone normality operator for $X$. Applying Theorem 2 we obtain a $\sigma$-disjoint open partial refinement $\mathcal{V}$ of $\mathcal{U}$ such that $X \backslash \cup \mathcal{V}$ is the union of a closed discrete collection $\mathcal{Y}$ of copies of stationary subsets of regular uncountable ordinals. Say $\mathcal{V}=\bigcup_{i \in \omega} \mathcal{V}_{i}$ where each $\mathcal{V}_{i}$ is a disjoint family of open sets each contained in a member of $\mathcal{U}$. We claim that it is sufficient to prove that there is a discrete $B \subseteq D$ such that $\bigcup \mathcal{Y} \subseteq S t(B, \mathcal{U})$ since then we proceed as follows. 
Since monotonically normal spaces are countably paracompact, if we define $V_{i}=$ $\cup \mathcal{V}_{i}$ for $i \in \omega$ and $V_{\omega}=S t(B, \mathcal{U})$, there is a locally finite open cover of $X,\left\{W_{i}\right.$ : $i \leq \omega\}$, such that $\overline{W_{i}} \subseteq V_{i}$.

Let

$$
P=\left\{\langle i, V\rangle: i \in \omega, V \in \mathcal{V}_{i}, V \cap W_{i} \neq \emptyset\right\} .
$$

For each $\langle i, V\rangle \in P$ choose $d(i, V) \in V \cap W_{i} \cap D$. For a fixed $i, E_{i}=\{d(i, V)$ : $\langle i, V\rangle \in P\}$ is discrete in $X$ (by the disjointness of $\mathcal{V}_{i}$ ) and therefore $E=\bigcup_{i \in \omega} E_{i}$ is also discrete in $X$ as a locally finite family of discrete sets in $X$. It is clear that $S t(B \cup E, \mathcal{U})=X$ and the theorem is proved.

To prove the existence of the set $B$ as claimed above we introduce the following notation.

Definition 2. If $Y \subseteq X$, then we say that $Y$ is $O K$ if $Y$ is closed in $X$ and for every neighborhood $N$ of $Y$ there is $B \subseteq D \cap N$ such that $B$ is discrete in $X$ and $Y \subseteq S t(B, \mathcal{U})$.

It is clear to see that it is sufficient to prove that $\bigcup \mathcal{Y}$ is OK. We prove this via the following lemmas.

Lemma 1. Every union of a closed discrete family of $O K$ sets in $X$ is $O K$.

Proof. Suppose $Y=\bigcup_{\alpha<\kappa} Y_{\alpha}$ where $\left\{Y_{\alpha}: \alpha<\kappa\right\}$ is closed and discrete in $X$, each $Y_{\alpha}$ is $\mathrm{OK}$ and $N$ is an open neighborhood of $Y$ in $X$. Since monotonically normal spaces are collectionwise normal, for each $\alpha<\kappa$ we can find an open $N_{\alpha} \subseteq N$ such that $Y_{\alpha} \subseteq N_{\alpha}$ and $\left\{\overline{N_{\alpha}}: \alpha<\kappa\right\}$ is closed discrete in $X$. Since each $Y_{\alpha}$ is OK, we can find $B_{\alpha} \subseteq N_{\alpha} \cap D$ such that $B_{\alpha}$ is discrete in $X$ and $Y_{\alpha} \subseteq \operatorname{St}\left(B_{\alpha}, \mathcal{U}\right)$. Then $B=\bigcup_{\alpha<\kappa} B_{\alpha}$ testifies to $Y$ being OK.

Lemma 2. If $Y \subseteq X$ is closed and homeomorphic to a subset of an ordinal $\kappa$, then $Y$ is $O K$.

Proof. Assume otherwise. Then there is a minimal $\kappa$ for which there is a non-OK closed $Y \subseteq X$ which is homeomorphic to a subset of $\kappa$. By Lemma 1 and the minimality of $\kappa, \kappa$ has uncountable cofinality and $Y$, identified with the required homeomorphic subset of $\kappa$ with the order of $\kappa$, is stationary in $\kappa$.

Before we complete the proof of Lemma 2 we need:

Lemma 3. If $Z \subseteq Y$ is $O K$ and (closed and) unbounded in $Y$, then $Y$ is $O K$.

Proof. Suppose we have such a $Z$. Then, if $N$ is a neighborhood of $Y$, there is $C \subseteq$ $(N \cap D)$ which is discrete in $X$ and such that $Z \subseteq S t(C, \mathcal{U})$. For $y \in(Y \backslash S t(C, \mathcal{U}))$, let

$$
W_{y}=\{w \in(Y \backslash S t(C, \mathcal{U})) \text { : there is no term of } Z \text { between } y \text { and } w\} .
$$

Observe that $W_{y}=W_{x}$ for all $x \in W_{y}$ and $\left\{W_{y}: y \in(Y \backslash S t(C, \mathcal{U}))\right\}$ is a closed discrete family of sets each of which is OK since $Z$ is unbounded and $\kappa$ is minimal. Hence, by Lemma 1 , there is $B \subseteq(N \cap D)$ which is discrete in $X$ with $(Y \backslash S t(C, \mathcal{U})) \subseteq S t(B, \mathcal{U})$. Then $B \cup C$ testifies to the OKness of $Y$, and this proves Lemma 3.

Returning to the proof of Lemma 2, let

$$
Y^{*}=\{y \in Y: y \in \operatorname{St}(\{x \in Y: x>y\}, \mathcal{U})\} .
$$


For each $y \in Y^{*}$ there is a minimal $y^{*} \in Y$ with $y^{*}>y$ and $y, y^{*} \in U_{y}$ for some $U_{y} \in \mathcal{U}$. Choose a closed unbounded $Z \subseteq Y$ such that $z \in\left(Z \cap Y^{*}\right)$ implies $z^{*}$ precedes the smallest element of $Z$ greater than $z$. We use the fact that $Y$ is unbounded in $\kappa$ to construct $Z$. By Lemma 3, to prove Lemma 2 it suffices to prove the following:

Lemma 4. $Z$ is $O K$.

For $y \in Y$ define $V_{y}=H(\{x \in Y: x \leq y\}, X \backslash\{x \in Y: x>y\})$. For $z \in Z \backslash Y^{*}$ arbitrarily choose $U_{z} \in \mathcal{U}$ with $z \in U_{z}$ (we have already chosen $U_{z}$ for $z \in Y^{*}$ ).

By induction, for each $\alpha<\kappa$, we choose $z_{\alpha} \in Z$ and $d_{\alpha} \in D \cap N$ as follows. For $\alpha \leq \kappa$ let $D_{\alpha}=\left\{d_{\beta}: \beta<\alpha\right\}$.

Let $z_{\alpha}$ be the first point of $Z \backslash S t\left(D_{\alpha}, \mathcal{U}\right)$ unless $Z \subseteq S t\left(D_{\alpha}, \mathcal{U}\right)$, in which case take $z_{\alpha}=z_{0}$. Then,

(i): if $z_{\alpha} \in Y^{*}$, choose $d_{\alpha} \in D \cap N \cap\left(U_{z_{\alpha}} \backslash \overline{V_{z_{\alpha}}}\right) \cap H\left(z_{\alpha}^{*}, V_{z_{\alpha}^{*}}\right)$,

(ii): if $z_{\alpha} \notin Y^{*}$, choose $d_{\alpha} \in D \cap N \cap V_{z_{\alpha}} \cap H\left(z_{\alpha}, H\left(z_{\alpha}, U_{z_{\alpha}}\right)\right)$,

(iii): if $\alpha>0$ and $z_{\alpha}=z_{0}$, take $d_{\alpha}=d_{0}$.

Claim. $D_{\kappa}$ is a subset of $D \cap N$ such that $Z \subseteq S t\left(D_{\kappa}, \mathcal{U}\right)$ and $D_{\kappa}$ is discrete in $X$.

Once we have proved this claim we will have completed the proof of Lemma 4 and hence that of Lemma 2 also.

Certainly $D_{\kappa} \subseteq D \cap N$. If $Z \nsubseteq S t\left(D_{\kappa}, \mathcal{U}\right)$ we have $Z \nsubseteq S t\left(D_{\alpha}, \mathcal{U}\right)$ for any $\alpha<\kappa$. However, then $z_{\alpha}$ is always defined as the first point of $Z$ not in $\operatorname{St}\left(D_{\alpha}, \mathcal{U}\right)$ and $z_{\alpha} \in S t\left(D_{\alpha+1}, \mathcal{U}\right)$. Thus $\left\{z_{\alpha}: \alpha<\kappa\right\}$ is a strictly increasing sequence in $\kappa$ with $z_{\alpha} \geq \alpha$ in $\kappa$ and therefore every term of $Z$ is in $\operatorname{St}\left(D_{\alpha}, \mathcal{U}\right)$ for some $\alpha$. Hence, $Z \subseteq S t\left(D_{\kappa}, \mathcal{U}\right)$, a contradiction.

To prove that $D_{\kappa}$ is discrete, suppose on the contrary that $\sigma \leq \kappa$ is minimal for $D_{\sigma}$ to have a limit point $x$. By the minimality of $\sigma, \sigma$ is a limit ordinal and $z_{\alpha} \neq z_{0}$ for all $\alpha$ with $0<\alpha<\sigma$.

Let $A^{*}=\left\{\alpha<\sigma: z_{\alpha} \in Y^{*}\right\}$ and $A=\left\{\alpha<\sigma: z_{\alpha} \notin Y^{*}\right\}$. Then $x$ is either a limit point of $\left\{d_{\alpha}: \alpha \in A^{*}\right\}$ or of $\left\{d_{\alpha}: \alpha \in A\right\}$.

Case 1. $x$ is a limit point of $\left\{d_{\alpha}: \alpha \in A^{*}\right\}$. By our choice of $Z$, if $\alpha<\beta$ in $A^{*}$, then $z_{\alpha}<z_{\alpha}^{*}<z_{\beta}<z_{\beta}^{*}$ and thus $V_{z_{\alpha}} \subseteq V_{z_{\alpha}^{*}} \subseteq V_{z_{\beta}} \subseteq V_{z_{\beta}^{*}}$. Since $d_{\beta} \notin V_{z_{\beta}}$, we have that $d_{\beta} \notin V_{z_{\alpha}^{*}}$. Consequently, by the minimality of $\sigma, x \notin V_{z_{\alpha}^{*}}$ for any $\alpha \in A^{*}$.

So if $x \in Y$, then $\left\{z_{\alpha}: \alpha \in A^{*}\right\}$ converges to $x$ as $\alpha$ increases. This follows since $x \notin V_{z_{\alpha}^{*}}$ implies that $x>z_{\alpha}^{*}$ for all $\alpha$ and if $\lim \left(z_{\alpha}\right)=\lambda<x$, then $H(x, X \backslash\{y \in$ $Y: y \leq \lambda\}) \cap H\left(z_{\alpha}^{*}, V_{z_{\alpha}^{*}}\right)=\emptyset$ for each $\alpha$ (by (MN3)) contradicting $x$ being a limit point of $\left\{d_{\alpha}: \alpha \in A^{*}\right\}$ since $d_{\alpha} \in H\left(z_{\alpha}^{*}, V_{z_{\alpha}^{*}}\right)$ for $\alpha \in A^{*}$.

Now $x \in U$ for some $U \in \mathcal{U}$ and hence there are $\alpha<\beta$ in $A^{*}$ with $d_{\alpha} \in U$ and $z_{\beta} \in U$ but this contradicts $z_{\beta} \notin S t\left(D_{\beta}, \mathcal{U}\right)$. Thus $x \notin Y$.

Hence there is $\alpha \in A^{*}$ with $d_{\alpha} \in H(x, X \backslash Y)$. However, $d_{\alpha}$ is also contained in $H\left(z_{\alpha}^{*}, V_{z_{\alpha}^{*}}\right)$ and since $z_{\alpha}^{*} \in Y$ and $x \notin V_{z_{\alpha}^{*}}$, this contradicts (MN3).

Case 2. $x$ is a limit point of $\left\{d_{\alpha}: \alpha \in A\right\}$. Thus, $A$ is cofinal in $\sigma$ and, since $\left\{z_{\alpha}: \alpha \in A\right\}$ is an increasing sequence in $Z$ as $\alpha$ increases to $\sigma$, it has a limit $\lambda \leq \kappa$.

Suppose $x \in Y$. Since $d_{\alpha} \in V_{z_{\alpha}}$ for $\alpha \in A, x \leq \lambda$. This follows since otherwise, $H(x, X \backslash\{y \in Y: y \leq \lambda\}) \cap H\left(z_{\alpha}, V_{z_{\alpha}}\right)=\emptyset$ for each $\alpha$ which contradicts $x$ being a limit point of $\left\{d_{\alpha}: \alpha \in A\right\}$. If $x=\lambda$, then the $z_{\alpha}$ for $\alpha \in A$ converge to $x$, and, as in case (1), choosing a $U \in \mathcal{U}$ with $x \in U$, there are $\alpha<\beta$ in $A$ with $d_{\alpha} \in U$ 
and $z_{\beta} \in U$, which again contradicts our choice of $z_{\beta} \notin S t\left(D_{\beta}, \mathcal{U}\right)$. So, $x<\lambda$. By the minimality of $\sigma$, if we let $Y^{\prime}=\{y \in Y: x<y\}$, then $x$ is a limit point of $\left\{d_{\alpha}: \alpha \in A\right.$ and $\left.z_{\alpha} \in Y^{\prime}\right\}$.

This defines $Y^{\prime}$ in the case $x \in Y$. If $x \notin Y$, then define $Y^{\prime}=Y$. In both cases $x$ is not in the interval $Y^{\prime}$ of $Y$ but $x$ is a limit point of $\left\{d_{\alpha}: \alpha \in A, z_{\alpha} \in Y^{\prime}\right\}$. Thus we can choose $\alpha<\beta$ in $A$ with $z_{\alpha}, z_{\beta} \in Y^{\prime}$ and $d_{\alpha}, d_{\beta} \in H\left(x, X \backslash Y^{\prime}\right)$.

Since $d_{\alpha} \in U_{z_{\alpha}}$, this ensures that $U_{z_{\alpha}} \subseteq S t\left(D_{\beta}, \mathcal{U}\right)$ and thus $z_{\beta} \notin U_{z_{\alpha}}$. Since $z_{\alpha} \notin$ $Y^{*}, z_{\alpha} \notin S t\left(z_{\beta}, \mathcal{U}\right)$; so $z_{\alpha} \notin U_{z_{\beta}}$. Therefore, by (MN3), $H\left(z_{\alpha}, U_{z_{\alpha}}\right) \cap H\left(z_{\beta}, U_{z_{\beta}}\right)=\emptyset$ and $x$ cannot belong to both. Say $x \notin H\left(z_{\alpha}, U_{z_{\alpha}}\right)$. But $d_{\alpha} \in H\left(x, X \backslash Y^{\prime}\right)$ and $z_{\alpha} \in Y^{\prime}$, while $d_{\alpha} \in H\left(z_{\alpha}, H\left(z_{\alpha}, U_{z_{\alpha}}\right)\right)$ which again contradicts (MN3). The proof of Lemma 4 is thus complete as is the proof of the theorem.

Theorem 3. Almost 2-fully normal spaces satisfy (a). In particular, every countably compact almost 2-fully normal space is acc.

Proof. Let $X$ be almost 2-fully normal and assume $\mathcal{U}$ is an open cover of $X$ and $D$ is a dense subset of $X$. Let $\mathcal{V}$ be an almost 2-star refinement of $\mathcal{U}$. Choose $d_{0}$ arbitrarily in $D$ and inductively choose $d_{\alpha} \in D$ such that $d_{\alpha} \notin S t\left(\left\{d_{\beta}: \beta<\alpha\right\}, \mathcal{V}\right)$. This process stops at some stage $\kappa$ and we have a set $F=\left\{d_{\alpha}: \alpha<\kappa\right\}$. We claim that $F$ is discrete in $X$ and $S t(F, \mathcal{U})=X$, and hence $X$ satisfies (a).

To show $F$ is discrete take any point $x \in X$ and choose $V \in \mathcal{V}$ such that $x \in V$. Assume for a contradiction that $d_{\alpha}, d_{\beta} \in V$ for $\beta<\alpha$. However this contradicts the choice of $d_{\alpha} \notin S t\left(\left\{d_{\beta}: \beta<\alpha\right\}, \mathcal{V}\right)$.

It is clear from the construction of $F$ that $D \subseteq S t(F, \mathcal{V})$ since otherwise we could have continued the choice of the $d_{\alpha}$ further. Take $x \in X$. Then $x \in V$ for some $V \in \mathcal{V}$. Since $D$ is dense there is some $d \in D \cap V$. Choose $d_{\alpha} \in F$ such that $d \in S t\left(d_{\alpha}, \mathcal{V}\right)$. We therefore have $x, d_{\alpha} \in S t(d, \mathcal{V})$ and by almost 2-full normality, there is $U \in \mathcal{U}$ such that $x, d_{\alpha} \in U$. Consequently we have proved that $X=S t(F, \mathcal{U})$.

\section{OTHER PROPERTIES}

The following result answers Question 1.13 in [14].

Theorem 4. Every countably compact, radial $T_{2}$-space is absolutely countably compact.

Proof. Let $X$ be a countably compact, radial $T_{2}$-space. If $X$ is not acc, then there exists an open cover $\mathcal{U}$ and a dense set $D$ such that for every countable $F \subseteq D$, we have $\operatorname{St}(F, \mathcal{U}) \neq X$. First we note for any $Y \subseteq X$ there exists a finite $F \subseteq Y$ such that $Y \subseteq S t(F, \mathcal{U})$ (in the case $Y=X$, we have the characterization of countable compactness that motivated the definition of acc (see [9, 3.12.23(d)] and [13])). In particular, there exists a finite set $F \subseteq[D]_{\aleph_{0}}$ such that $[D]_{\aleph_{0}} \subseteq S t(F, \mathcal{U})$. It follows (as in the proof of [13, Theorem 1.8]) that there exists a countable $G \subseteq D$ such that $[D]_{\aleph_{0}} \subseteq S t(G, \mathcal{U})$. Now we construct, by induction, points $x_{n} \in X$ and sets $U_{n} \in \mathcal{U}$ satisfying the following conditions:

(i): $x_{n} \in U_{n}$,

(ii): $x_{n} \notin S t(G, \mathcal{U}) \cup \bigcup_{j<n} U_{j}$.

Let $x$ be a complete accumulation point of the infinite set $\left(x_{n}\right)$. Clearly $x \neq x_{n}$ for all $n$; so by $T_{2}$ there exists an open set $V_{n}$ such that $x_{n} \in V_{n}$ and $x \notin \overline{V_{n}}$. 
Since $x$ is a limit point of $\left\{x_{n}: n<\omega\right\}$ and $D$ is dense, we have that $x$ is in the closure of $Y=\bigcup_{n \in \omega}\left(V_{n} \cap D\right)$. Since $X$ is radial, there exists a transfinite sequence $f: \kappa \rightarrow Y$ such that $f$ converges to $x$. We claim that $k=\omega:$ If not, then put $A_{n}=\left\{\alpha<\kappa: f(\alpha) \in V_{n}\right\}$. Since $\kappa$ is regular uncountable, there exists $n<\omega$ such that $\left|A_{n}\right|=\kappa$. Since $f$ converges to $x$, every neighborhood of $x$ contains the image under $f$ of a final segment of $\kappa$; hence of $A_{n}$. But this is impossible since $f\left(A_{n}\right) \subseteq V_{n}$ and $x \notin \overline{V_{n}}$. Thus $\kappa=\omega$, and therefore $x \in[D]_{\aleph_{0}} \subseteq \operatorname{St}(G, \mathcal{U})$. Thus $\operatorname{St}(G, \mathcal{U})$ is an open neighborhood of $x$ which does not contain any $x_{n}$, contradicting the fact that $x$ is an accumulation point of the $x_{n}$.

A standard example of a countably compact space that is not acc is the product of ordinals $\omega_{1} \times\left(\omega_{1}+1\right)$ [13]. This space is known, and easily shown, to be pseudoradial (even semi-radial [6], and R-monolithic [5]). Thus the hypothesis of "radial" in the preceding theorem cannot be weakened to "pseudoradial".

We conclude our discussion of radial spaces by noting that there exists a pseudocompact radial space which does not have property (a). The space is $\Psi(\mathcal{A})$ where $\mathcal{A}$ is an infinite maximal almost disjoint family of infinite subsets of $\omega[9,3.6$.I(a) $]$ (this example was also noted by Matveev). To prove that $\Psi(\mathcal{A})$ does not satisfy property (a), pick distinct $A_{n} \in \mathcal{A}$ for $n \in \omega$, and take $D=\omega$ and

$$
\mathcal{U}=\left\{\left(A_{n} \backslash n\right) \cup\left\{x_{A_{n}}\right\}: n \in \omega\right\} \cup\left\{A \cup\left\{x_{A}\right\}: A \in \mathcal{A} \text { and } A \neq A_{n} \forall n \in \omega\right\} \cup \omega .
$$

If $E \subseteq \omega$ is closed discrete, then $E$ is finite, and thus there exists $n \in \omega$ such that $\left(A_{n} \backslash n\right) \cap E=\emptyset$. Hence the point $x_{A_{n}} \notin S t(E, \mathcal{U})$. The space $\Psi(\mathcal{A})$ is first countable (hence radial).

We end this section with

Theorem 5. Every countably compact $T_{3}$-space with countable spread is acc.

This follows immediately from two known results. A. Arhangel'skiü [3, Corollary 3] proved that every countably compact $T_{3}$-space with countable spread has countable tightness, and Matveev [13, Theorem 1.8] proved that every countably compact space with countable tightness is acc.

\section{Relations between Several of these properties}

It is well known that generalized ordered spaces (GO-spaces) are monotonically normal [11] (thus satisfy (a)), orthocompact [10, 5.23 and p. 190], almost 2-fully normal [12], and radial. These properties provide five reasons why countably compact GO-spaces are acc. Figure 1 indicates all the implications that hold between pairs of these six properties (examples to show that in general no other implications that hold can be found readily in the literature).

Theorem 6 below gives a relation among three of these properties.

Definition 3 ([12]). A space $X$ is said to be finitely fully normal if for every open cover $\mathcal{U}$ of $X$ there is an open refinement $\mathcal{V}$ of $\mathcal{U}$ such that if $x \in X$ and $\mathcal{V}_{0}$ is a finite subcollection of $\mathcal{V}$ with $x \in \bigcap \mathcal{V}_{0}$, then there is a $U \in \mathcal{U}$ such that $\bigcup \mathcal{V}_{0} \subseteq U$.

Theorem 6. A monotonically normal, orthocompact space is finitely fully normal (and therefore, in particular, almost 2-fully normal).

Proof. Let $H$ be an MN operator for $X$. Assume $\mathcal{U}$ is an open cover of $X$. By orthocompactness, there exists an open refinement $\mathcal{W}$ of $\mathcal{U}$ such that for each $x \in X$, the set $W_{x}=\bigcap\{W \in \mathcal{W}: x \in W\}$ is open. Note that, if $x \in W_{y}$, then $W_{x} \subseteq W_{y}$. 


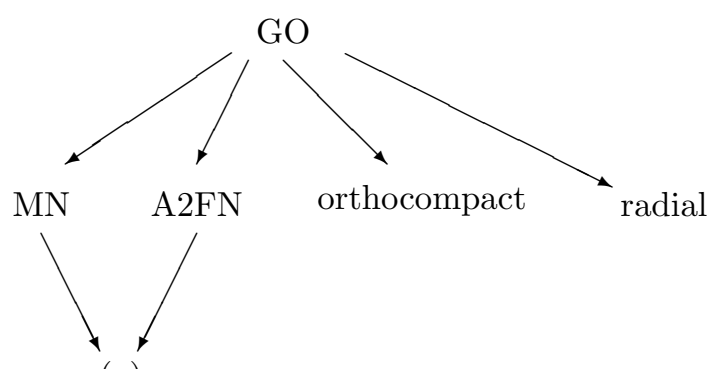

(a)

Figure 1

Let $\mathcal{V}=\left\{H\left(x, W_{x}\right): x \in X\right\}$. We claim that $\mathcal{V}$ testifies to the fact that $X$ is finitely fully normal.

Assume $x \in H\left(z_{i}, W_{z_{i}}\right)$ for $i \leq n$. By (MN3), for all $i, j \leq n$, either $z_{i} \in W_{z_{j}}$ or $z_{j} \in W_{z_{i}}$ and therefore either $W_{z_{i}} \subseteq W_{z_{j}}$ or $W_{z_{j}} \subseteq W_{z_{i}}$. Consequently, $\left\{W_{z_{i}}: i \leq\right.$ $n\}$ is linearly ordered by inclusion and hence there is $j \leq n$ such that $W_{z_{i}} \subseteq W_{z_{j}}$ for all $i \leq n$. Since $\mathcal{W}$ refines $\mathcal{U}$ there is $U \in \mathcal{U}$ with $W_{z_{j}} \subseteq U$ and therefore $\bigcup\left\{H\left(z_{i}, W_{z_{i}}\right): i \leq n\right\} \subseteq U$.

We do not know if the conclusion of the preceding theorem can be strengthened to $\aleph_{0}$-fully normal. The ordinal space $\omega_{1}$ is a GO-space that is not $\aleph_{1}$-fully normal (see [12]).

The authors wish to thank Michael Matveev for sharing his ideas with them, in particular the definition of property (a).

\section{REFERENCES}

[1] A. V. Arhangel'skiı̌, On bicompacta hereditarily satisfying the Souslin condition. Tightness and free sequences. Soviet Math. Dokl. 12 (1971), 1253-1257.

[2] A. V. Arhangel'skiı̌, Structure and classfication of topological spaces, Russian Math. Surveys 33 No. 5 (1978) 33-96.

[3] A. V. Arhangel'skiı̌, Countably compact groups, Math. Japonica 40 (1994) 39 - 53. MR 95i: 54001

[4] Z. Balogh and M. E. Rudin, Monotone normality, Topology Appl. 47 (1992) 115-127. MR 94b:54065

[5] Angelo Bella, Few remarks and questions on pseudo radial and related spaces, to appear.

[6] A. Bella and Gerlitz, On a condition for the pseudo radiality of a product, Comment. Math. Univ. Carolin. (Prague) 33 (1992) 311-313. MR 93h:54001

[7] C. J. R. Borges, A study of monotonically normal spaces, Proc. Amer. Math. Soc. 38 (1973) 211-214. MR 48:2994

[8] E. K. van Douwen, Simultaneous extension of continuous functions, in Eric K. van Douwen, Collected Papers, Volume 1, J. van Mill, ed., North-Holland, Amsterdam, 1994. MR 96a:01047

[9] Ryszard Engelking, General Topology, Heldermann Verlag, Berlin 1989. MR 91c:54001

[10] Peter Fletcher and William F. Lindgren, Quasi-uniform Spaces, Marcel Dekker, New York 1982. MR 84h:54026

[11] R. W. Heath, D. J. Lutzer, and P. L. Zenor, Monotonically normal spaces, Trans. Amer. Math. Soc. 178 (1973) 481-493. MR 51:9030

[12] M. J. Mansfield, Some generalizations of full normality, Trans. Amer. Math. Soc. 86 (1957) 489-505. MR 20:273

[13] Michael V. Matveev, Absolutely countably compact spaces, Topology Appl. 58 (1994) 81-92. MR 95e:54035 
[14] Jerry E. Vaughan, On the product of a compact space with an absolutely countably compact space, to appear in the Proceedings of the Vrije University Topology Conference, Amsterdam, 1994.

(Mary Ellen Rudin) Department of Mathematics, University of Wisconsin, Madison, WISCONSIN 53706

E-mail address: mrudin@math.wisc.edu

(Ian S. Stares and Jerry E. Vaughan) Department of Mathematical Sciences, University of North Carolina at Greensboro, Greensboro, North Carolina 27412

E-mail address: isstares@maths.ox.ac.uk

E-mail address: vaughanj@steffi.uncg.edu 